\title{
Structural effects on the hole mobilities of indenothiophene-embedded homologs
}

\author{
Teng-Chih Chao ${ }^{\mathrm{a}}$, Ken-Tsung Wong ${ }^{\mathrm{a}, *}$, Wen-Yi Hung ${ }^{\mathrm{b}, *}$, Tei-Hung Hou ${ }^{\mathrm{b}}$, Wei-Jiun Chen ${ }^{\mathrm{b}}$ \\ a Department of Chemistry, National Taiwan University, Taipei 106, Taiwan \\ ${ }^{\mathrm{b}}$ Institute of Optoelectronic Sciences, National Taiwan Ocean University, Keelung 202, Taiwan
}

\section{A R T I C L E I N F O}

\section{Article history:}

Received 7 January 2009

Revised 17 February 2009

Accepted 19 February 2009

Available online 25 February 2009

\begin{abstract}
A B S T R A C T
A systematic study of the relationship between the structures and the non-dispersive hole mobilities (up to $10^{-3} \mathrm{~cm}^{2} \mathrm{~V}^{-1} \mathrm{~s}^{-1}$ ) of a homologous series of amorphous indenothiophene-containing materials is described. The hole mobilities were dependent mainly on the length and rigidity of the $\pi$-conjugated backbone and the peripheral substituents.
\end{abstract}

(c) 2009 Elsevier Ltd. All rights reserved.
Recent developments in organic electronic and optoelectronic devices, such as organic light-emitting diodes (OLEDs), solar cells, electrochromic cells, and organic field effect transistors (OTFTs), have imposed substantial demands on organic semiconducting materials. Although such devices perform a diverse range of functions, charge carrier transport is a common key issue for each system, and its optimization remains a challenge for the development of practical or commercial devices. The charge transport behavior of amorphous organic materials can be described using Marcus theory ${ }^{1}$ :

$k_{\mathrm{et}}=\frac{2 \pi}{h} \frac{H_{\mathrm{da}}^{2}}{\sqrt{4 \pi \lambda k T}} \exp -\frac{\left(\Delta G^{0}+\lambda\right)^{2}}{4 \lambda k T}$

in which the rate of intermolecular electron hopping is governed by the reorganization energy $(\lambda)$ and the charge transfer integral $\left(H_{\mathrm{da}}\right)$, with the latter especially being the bottle-neck parameter. ${ }^{2}$ Therefore, the molecular design of organic $\pi$-conjugated charge carriertransporting materials that can reduce the reorganization energy and enhance the electronic coupling factor during electron hopping processes has emerged as an effective approach toward achieving novel materials exhibiting high mobility. In this regard, molecules that feature rigid, coplanar units are excellent candidates; ${ }^{3}$ for example, polyacenes, especially pentacene, exhibit remarkable carrier mobility. ${ }^{4}$ Unfortunately, non-homogeneous crystalline systems that feature grain boundaries of various sizes often lead to the generation of carrier traps, which may cause problems in device applications. Chemical modification of the coplanar core structure with sterically hindered peripheral substituents is an effective means of suppressing crystal formation, leading to amorphous materials that can form homogeneous thin films, while retaining sufficient charge transporting character. ${ }^{5}$ In this Letter, we report

\footnotetext{
* Corresponding authors. Tel.: +886 2 33661665; fax: +886 233661667 (K.-T.W). E-mail addresses: kenwong@ntu.edu.tw (K.-T. Wong), wenhung@mail.ntou. edu.tw (W.-Y. Hung).
}

a systematic study of the chemical structures and hole mobilities of a homologous series of amorphous indenothiophene-based $\pi$ conjugated materials (Scheme 1). Our results indicate that the hole mobilities are dependent mainly on the length and rigidity of the $\pi$ conjugated backbone, with the peripheral substituents playing a subtle role in governing the hole-transporting behavior. We observed hole mobilities of up to $10^{-3} \mathrm{~cm}^{2} \mathrm{~V}^{-1} \mathrm{~s}^{-1}$ for a rigid, coplanar, amorphous indenothiophene-based derivative bearing small, uniform peripheral aryl substituents.
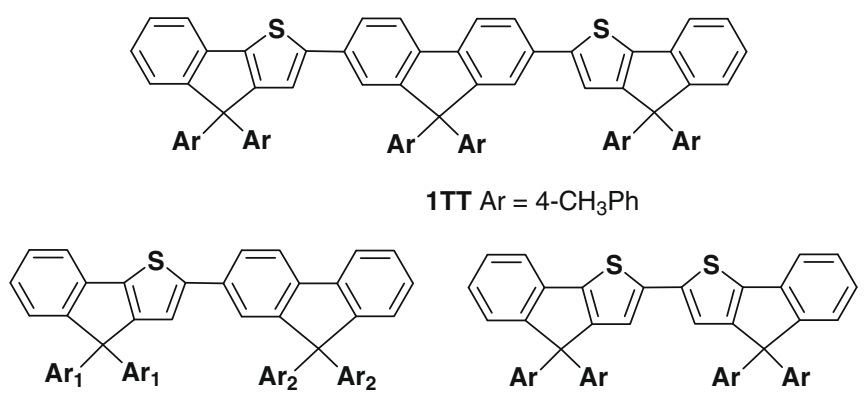

2TT $\mathrm{Ar}_{1}=4-\mathrm{CH}_{3} \mathrm{Ph}, \mathrm{Ar}_{2}=4-\mathrm{CH}_{3} \mathrm{Ph}$ 2PT $\mathrm{Ar}_{1}=\mathrm{Ph}, \mathrm{Ar}_{2}=4-\mathrm{CH}_{3} \mathrm{Ph}$

3PP $\mathrm{Ar}=\mathrm{Ph}$ 3TT $\mathrm{Ar}=4-\mathrm{CH}_{3} \mathrm{Ph}$

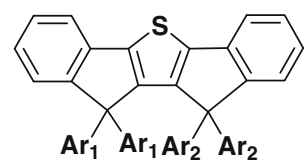

4PP $\mathrm{Ar}_{1}=\mathrm{Ph}, \mathrm{Ar}_{2}=\mathrm{Ph}$

4PT $\mathrm{Ar}_{1}=\mathrm{Ph}, \mathrm{Ar}_{2}=4-\mathrm{CH}_{3} \mathrm{Ph}$

4TT $\mathrm{Ar}_{1}=4-\mathrm{CH}_{3} \mathrm{Ph}, \mathrm{Ar}_{2}=4-\mathrm{CH}_{3} \mathrm{Ph}$

Scheme 1. Structures of indenothiophene-derived homologs. 
Oligothiophenes and their related derivatives are promising materials for use in carrier-transporting applications. ${ }^{6}$ However, conformational disorder of the $\pi$-conjugated backbone of thiophene-containing materials has the potential to reduce the intermolecular electronic coupling efficiency, which in turn limits the carrier mobility. We envisioned that the conformational flexibility of the molecular backbone would be reduced significantly through flattening of the thiophene moiety and adjacent arene ring(s) into a coplanar configuration. In our approach, one (or more) $\mathrm{sp}^{3}$-hybridized carbon atom bearing two aryl substituents would serve as a bridging unit flattening the molecular planes of the thiophene and phenylene moieties into coplanarity to form an indenothiophene skeleton, Scheme 1 illustrates the molecular structures of a series of indenothiophene-derived homologs used for this current work. For systematic investigation of the structure and mobility correlation, indenothiophene derivatives 2TT, 2PT, 3PP, and 3TT were synthesized (Scheme S1, supplementary data) in addition to previously reported $\mathbf{1 T T}$, 4PP, 4PT, and $\mathbf{4 T T}$. $^{7}$ The C2 position of indenothiophene can be readily functionalized and further transformed into a homologous series with structural features including (1) hybridization with a coplanar fluorene ring gives 1TT, 2TT, and 2PT; (2) homo-couplings of indenothiophenes afford 3PP and 3TT; and (3) sequential annulation with adjacent phenylene rings gives the rigid and coplanar 4PP, 4PT, and 4TT. The introduction of aryl groups as peripheral substituents provides high tolerance toward thermal decomposition (analyzed by TGA) and high morphological stability with distinct glass transition temperatures (analyzed by DSC) (Table S1, supplementary data). Thus, homogeneous thin films can be obtained through thermal evaporation under high vacuum.

We conducted charge carrier mobility measurements for the indenothiophene derivatives at ambient temperature using a time-of-flight (TOF) technique. Clear constant-current plateaus in typical photocurrent transients of the hole mobility measurements (Fig. S1, supplementary data) were observed, which are the signature of non-dispersive carrier transport behavior. The calculated field-dependent hole mobilities of the indenothiophene derivatives are plotted as a function of $\mathrm{E}^{1 / 2}$ in Figure 1 . We observed that the universal Poole-Frenkel relationship $\left[\mu \propto \exp \left(\beta \mathrm{E}^{1 / 2}\right)\right.$, where $\beta$ is the Poole-Frenkel factor] is followed. ${ }^{8}$ The hole mobilities exist in the range from $4 \times 10^{-5}$ to $10^{-3} \mathrm{~cm}^{2} \mathrm{~V}^{-1} \mathrm{~s}^{-1}$ for fields varying from $10^{5}$ to $6.7 \times 10^{5} \mathrm{~V} \mathrm{~cm}^{-1}$. In contrast, the TOF transient for electrons exhibited strong dispersive photocurrents.

The observed hole mobility of 1TT is comparable to, but slightly lower than that of its pure hydrocarbon counterpart, terfluorene T3, ${ }^{9}$ which we ascribe mainly to the relatively non-linear molecular backbone of 1TT resulting from the intrinsic molecular geometry of the thiophene ring. This deviation from linearity for 1TT

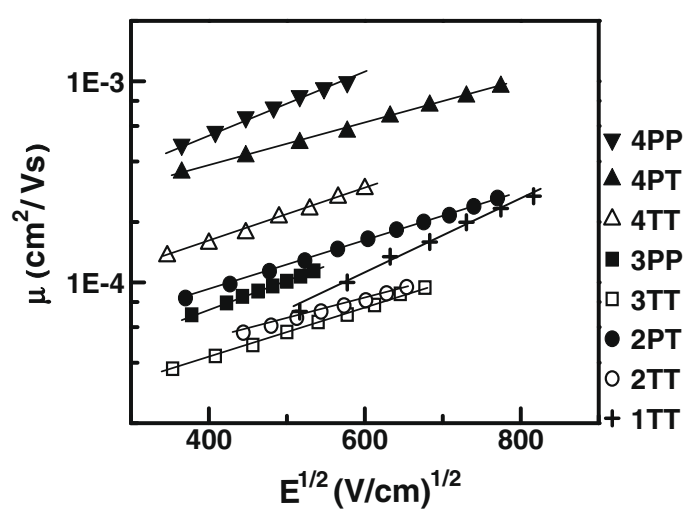

Figure 1. Hole mobilities plotted against $E^{1 / 2}$ for the indenothiophene-embedded homologs. increases the conformational flexibility, resulting in larger reorganization energy and, weaker intermolecular interactions, which could render carrier transport more difficult. Although the reduced molecular lengths of 2TT and 2TP could diminish the complexity of their molecular configurations, we detected no significant enhancements in their hole mobilities. The homo-coupled bis(indenothiophene)s, 3PP and 3TT, exhibit hole mobilities that are comparable to those of their counterparts 2PT and 2TT. These results indicate that hybridization of the characteristics of the coplanar fluorene and indenothiophene moieties did not provide any evident benefit to hole mobility. Figure 1 indicates that the rigid and coplanar homologs 4PP, 4PT, and 4TT exhibit remarkably improved hole mobilities (e.g., up to $10^{-3} \mathrm{~cm}^{2} \mathrm{~V}^{-1} \mathrm{~s}^{-1}$ ) at high electric field, making them competitive with typical arylamine-based hole transporting materials. ${ }^{10}$ This finding confirms the advantages of rigidity and coplanarity of the molecular backbone that contribute to the carrier transportation properties. Importantly, the structures of the aryl substituents exert a significant effect on the hole mobilities. Figure 2 depicts the hole mobilities plotted as functions of the different types of substituents attached to common indenothiophene-embedded backbones. The hole mobilities follow the orders 4PP > 4PT > 4TT, 2PT > 2TT, and 3PP > 3TT.

Molecular calculation of 4TT reveals an optimized ground state geometry that is consistent with the experimental one determined through the X-ray crystallographic analysis; ${ }^{7}$ the frontier molecular orbitals (HOMO and LUMO; Fig. 3) of 4TT are localized mainly on the rigid and coplanar indenothiophene backbone without a significant contribution from the peripheral tolyl substituents. The result of MO calculation is consistent with the nearly identical absorption and emission characteristics of molecules having the same indenothiophene backbone but bearing different substituents (Fig. S2, supplementary data). In addition, the reorganization energies of 4PP, 4PT, and 4TT upon hole-transportation were calculated at B3LYP/631G ${ }^{*}$ level with Jaguar 7.0 quantum chemistry software to be $270 \mathrm{meV}, 274 \mathrm{meV}$, and $270 \mathrm{meV}$, respectively. These results indicate that the aryl substituents on the top and bottom faces of the molecular plane behave only as effective spatial hindrance groups to suppress crystallization and improve morphological stability. Thus, the smaller substituents (i.e., phenyl groups) reduce the carrier hopping distance between the molecules, leading to improved mobility. ${ }^{11}$ For the same substituents (e.g., two ditoyl units), we found that the hole mobility of 4TT was over three times higher than those of 2TT and 3TT. The results again suggest that longer backbones lead to more disordered molecular conformations and, thus, lower hole mobilities.

In order to probe the effect of structural features that govern the hole mobilities, we also accomplished temperature and field-

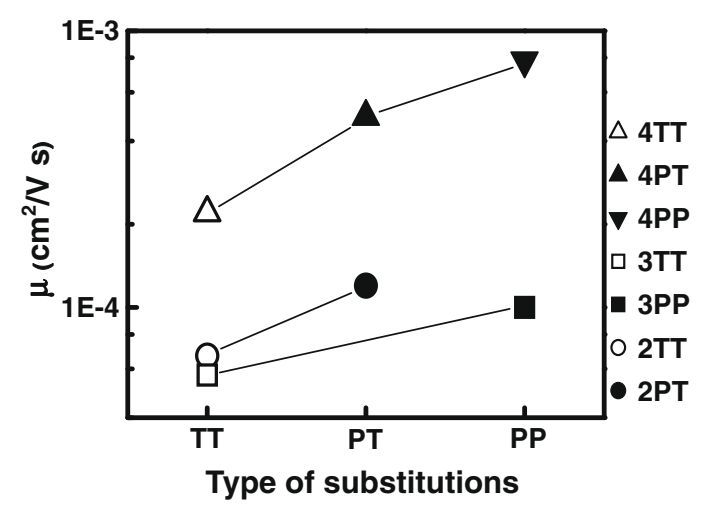

Figure 2. Hole mobilities (at $E=2.5 \times 10^{5} \mathrm{~V} \mathrm{~cm}^{-1}$ ) plotted with respect to the different types of substituents (TT: two ditoyl units; PT: one diphenyl and one ditoyl unit; PP: two diphenyl units) attached to common indenothiopheneembedded backbones. 


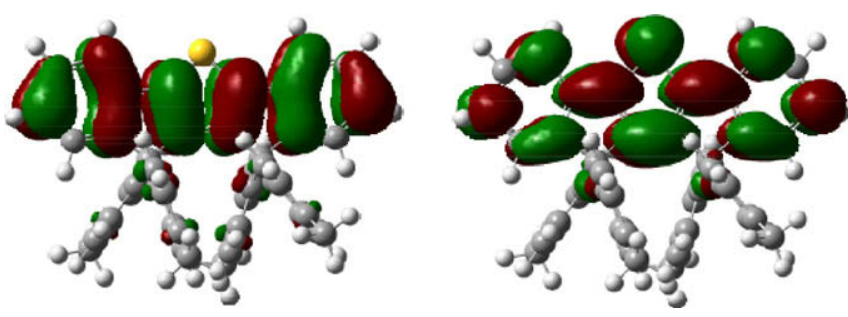

Figure 3. Frontier molecular orbitals HOMO (left) and LUMO (right) of 4TT computed using the DFT (B3LYP/631G) method.

Table 1

GDM carrier transport parameters for 4PP, 4PT, and 4TT

\begin{tabular}{lllll}
\hline Hole & $\mu_{0}\left(\times 10^{-3} \mathrm{~cm}^{2} / \mathrm{V} \mathrm{s}\right)$ & $\sigma\left(\times 10^{-2} \mathrm{eV}\right)$ & $\Sigma$ & $C\left(\times 10^{-4}\right)$ \\
\hline 4PP & 15 & 8.00 & 1.65 & 4 \\
4PT & 10 & 7.80 & 1.63 & 3.7 \\
4TT & 3 & 7.75 & 1.58 & 4.5 \\
\hline
\end{tabular}

dependent hole mobility measurements of 4PP, 4PT, and 4TT (Fig. S3, supplementary data). The obtained carrier mobilities agree with the prediction of Gaussian disorder model (GDM) $)^{8,12}$, which assumes that charge transport in amorphous organic solids occurs by means of hopping among uncorrelated localized sites with Gaussian-distributed energetic disorder and intermolecular positional disorder. Table 1 summarizes the extracted data: disorderfree mobility $\left(\mu_{0}\right)$, degree of energetic disorder $(\sigma)$, and the parameter of positional disorder $(\Sigma)$ from temperature- and fielddependent hole mobilities using Bässler formalism described as:

$\mu(E, T)=\mu_{0} \exp \left(-\left[\frac{2 \sigma}{3 k_{\mathrm{B}} T}\right]^{2}\right) \exp \left(C\left[\left(\frac{\sigma}{k_{\mathrm{B}} T}\right)^{2}-\Sigma^{2}\right] \sqrt{E}\right)$

where $E$ is electric field, $T$ is temperature, $k_{\mathrm{B}}$ is the Boltzmann constant, $C$ is an empirical constant.

The obtained data indicate that the nature of peripheral substitutions exhibits negligible influence on the energetic disorder, as well as on the positional disorder. More significantly, the results of temperature-dependent mobility measurement suggest that the relative magnitudes of carrier mobility of a common indenothiophene backbone with different substitutions are mainly governed by $\mu_{0}$ (4PP $>$ 4PT $>$ 4TT) (i.e., intrinsic intermolecular charge-transfer characteristics), which is consistent with those observed for mobilities.

In summary, TOF measurements of the non-dispersive hole mobilities of a homologous series of amorphous indenothioph- ene-based $\pi$-conjugated materials are reported first time, the results indicate that their hole mobilities are influenced strongly by the length and rigidity of their $\pi$-conjugated backbones and by the structures of the peripheral substituents. Hole mobilities of up to $10^{-3} \mathrm{~cm}^{2} \mathrm{~V}^{-1} \mathrm{~s}^{-1}$ were observed for a rigid, coplanar indenothiophene-based derivative (4PP) bearing small, uniform peripheral aryl substituents.

\section{Acknowledgments}

We thank Mr. Wei-Guang Liu for the aid in molecular calculation on the reorganization energy. The financial support of this research was from the National Science Council of Taiwan.

\section{Supplementary data}

Supplementary data associated with this article can be found, in the online version, at doi:10.1016/j.tetlet.2009.02.148.

\section{References and notes}

1. (a) Marcus, R. A. Rev. Mod. Phys. 1993, 65, 599; (b) Cornil, J.; Beljonne, D.; Calbert, J.-P.; Brédas, J.-L. Adv. Mater. 2001, 13, 1053.

2. (a) Brédas, J. L.; Calbert, J. P.; da Silvo Filho, D. A.; Cornil, J. Proc. Natl. Acad. Sci. U.S.A. 2002, 99, 5804; (b) Lin, B. C.; Cheng, C. P.; You, Z.-Q.; Hsu, C.-P. J. Am. Chem. Soc. 2005, 127, 66

3. (a) Yamaguchi, S.; Xu, C.; Okamoto, T. Pure Appl. Chem. 2006, 78, 721; (b) Hertel D.; Scherf, U.; Bässeler, H. Adv. Mater. 1998, 10, 1119; (c) Walkim, S.; Bouchard, J.; Simard, M.; Drolet, N.; Tao, Y.; Leclerc, M. Chem. Mater. 2004, 16, 4386; (d) Li, Y.; Wu, Y.; Gardner, S.; Ong, B. S. Adv. Mater. 2005, 17, 849.

4. (a) Nelson, S. F.; Lin, Y.-Y.; Gundlach, D. J.; Jackson, T. N. Appl. Phys. Lett. 1998 72, 1854; (b) Lee, J. Y.; Roth, S.; Park, Y. W. Appl. Phys. Lett. 2006, 88, 252106.

5. (a) Anthony, J. E.; Brooks, J. S.; Eaton, D. L.; Parkin, S. R. J. Am. Chem. Soc. 2001, 123, 9482; (b) Jiang, J.; Kaafarani, B. R.; Neckers, D. C. J. Org. Chem. 2006, 71 2155.

6. (a) Yoon, M.-H.; Facchetti, A.; Stern, C. E.; Marks, T. J. J. Am. Chem. Soc. 2006, 128 5792; (b) Murphy, A. R.; Chang, P. C.; VanDyke, P.; Liu, J.; Fréchet, J. M. Subramanian, V.; DeLongchamp, D. M.; Sambasivan, S.; Fischer, D. A.; Lin, E. K. Chem. Mater. 2005, 17, 6033; (c) Meng, H.; Bao, Z.; Lovinger, A. J.; Wang, B.-C.; Mujsce, A. M. J. Am. Chem. Soc. 2001, 123, 9214

7. (a) Wong, K.-T.; Hwu, T.-Y.; Balaiah, A.; Chao, T.-C.; Fang, F.-C.; Lee, C.-T.; Peng Y.-C. Org. Lett. 2006, 8, 1415; (b) Wong, K.-T.; Chao, T.-C.; Chi, L.-C.; Chu, Y.-Y.; Balaiah, A.; Chiu, S.-F.; Liu, Y.-H.; Wang, Y. Org. Lett. 2006, 8, 5033.

8. (a) Bässler, H. Philos. Mag. B 1992, 65, 795; (b) Bässler, H. Phys. Status Solidi B 1993, 175, 15; (c) Borsenberger, P. M.; Pautmeier, L.; Bässler, H. J. Chem. Phys. 1991, 94, 5447 .

9. Wu, C.-C.; Liu, T.-L.; Hung, W.-Y.; Lin, Y.-T.; Wong, K.-T.; Chen, R.-T.; Chen, Y.M.; Chien, Y.-Y. J. Am. Chem. Soc. 2003, 125, 3710.

10. (a) Strohriegl, P. Adv. Mater. 2002, 14, 1439; (b) Shirota, Y. J. Mater. Chem. 2000 $10,1$.

11. (a) Wu, C.-C.; Liu, W.-G.; Hung, W.-Y.; Liu, T.-L.; Lin, Y.-T.; Lin, H.-W.; Wong, K.T.; Chien, Y.-Y.; Chen, R.-T.; Hung, T.-H.; Chao, T.-C.; Chen, Y.-M. Appl. Phys. Lett. 2005, 87, 052103; (b) Fong, H. H.; So, S. K.; Sham, W. Y.; Lo, C. F.; Wu, Y. S.; Chen, C. H. Chem. Phys. 2004, 298, 119.

12. (a) Poplavskyy, D.; Nelson, J. J. Appl. Phys. 2003, 93, 341; (b) Laquai, F.; Wegner, G.; Bässler, H.; Heun, S. J. Appl. Phys. 2006, 99, 203712. 